\title{
Intra-Car Wireless Sensor Networks Using RFID: Opportunities and Challenges
}

\author{
Ozan K. Tonguz \\ tonguz@ece.cmu.edu \\ Hsin-Mu Tsai \\ Carnegie Mellon University, ECE Department \\ Pittsburgh, PA 15213-3890, USA
}

\author{
Cem Saraydar \\ cem.saraydar@gm.com
}

\author{
Timothy Talty \\ timothy.talty@gm.com andrew.macdonald@gm.com \\ General Motors Corporation \\ Warren, MI 48092-2031, USA
}

\begin{abstract}
Building a wireless sensor network in a car has important benefits in terms of cost reduction, having an open architecture, and fuel efficiency. One option for implementing such an intra-car wireless network is to use RFID technology. In this paper, we report the results of extensive experiments carried out at Carnegie Mellon University in collaboration with General Motors for understanding the capabilities and limitations of RFID technology.
\end{abstract}

\section{INTRODUCTION}

In modern cars the sensors are connected to the central unit (microprocessors) with wires. As the number of sensors in cars keep increasing every year, it will be important to eliminate these wires and connect the sensors to microprocessors in a wireless fashion. Building a wireless sensor network in a car has important advantages in terms of cost reduction, having an open architecture, and fuel efficiency. One option for implementing such an intra-car wireless network is to use RFID technology. In this paper, we report the results of extensive experiments carried out at Carnegie Mellon University in the last 2 years in collaboration with General Motors for understanding the capabilities and limitations of RFID technology. We show that while RFID technology is an interesting and attractive option for building an intra-car wireless sensor network, several technical problems need to be resolved for making it a feasible solution for this interesting application.

\section{Problem Statement}

The current architecture of the sensor network in a car is shown in Figure 1(a). Each sensor is connected to the serial data bus via an adapter. The sensor information is relayed to the serial data bus, and then sent to the embeded microprocessor in the car for further processing. The operating power of the sensors is supplied by the car battery via the power distribution network. The current wired architecture has the following disavantages: (i) The vast amount of wiring shrinks the layout space and decrease the serviceability and reliability [1]; (ii) the cost of the data and power wires for the sensors and the manufacturing and assembly operations could cost up to hundreds of dollars; (iii) the wires increase the weight of the car and decrease the fuel efficiency [2]; and (iv) sensors cannot be installed in some of the locations (tires, steering wheel, etc.) because they cannot be reached by wires.
One solution to this problem could be the use of RFID technology whereby the wires from sensors to the serial data bus are replaced by a Reader and several Tags (Transponders) that will be connected to the sensors. Given that passive RFID Tags are extremely cheap (a few cents each), the main expense of this solution is the Reader which could cost several tens of dollars. In one possible configuration, the Reader periodically obtains the measurement made by sensors by sending an energizing pulse to each Tag. The Tag uses the energizing pulse to subsequently turn on and then transmits the data it has collected by using a portion of this energy. The envisioned scenario is illustrated in Figure 1(b).

\section{EXPERIMENTAL METHOD AND RESUlts}

To understand the physical characteristics of the wireless channels between a Reader and a Tag, the position of the Reader (transmitter) was fixed to a location above the driver's seat (See Figure 2(a)). By placing the RFID Tags (receivers) into different locations; categorically, four different communication channels were identified inside a car: (i) channel to/from Tags on the hood $(\mathrm{H})$; (ii) channel to/from Tags in the trunk (TR); (iii) channel to/from Tags inside the engine compartment (IE) (Figure 2(b)); and (iv) channel to/from Tags under the engine compartment (UE) (Figure 2(c)). The following experiments were carried out in a General Motors '96 Buick LaSabre car at Carnegie Mellon University.

\section{A. Power Delay Profile Measurements}

The purpose of this measurement was to understand the severity of the multipath effect in the car environment. The power delay profiles (PDF) [3] of all four channels were measured using the vector network analyzer and two $5 \mathrm{dBi}$ omni-directional antennas as shown in Figure 3. The configuration parameters are shown in Table I. Coherence bandwidths were then computed using the power delay profiles obtained. We used the definition of the coherence bandwidth as the bandwidth over which the frequency correlation function is above $50 \%$, given by

$$
B=\frac{1}{5 \sigma_{\tau}}
$$

where $B$ is the coherence bandwidth and $\sigma_{\tau}$ is the root mean square (RMS) delay spread of the channel. The data points representing the noise were excluded in the calculation of 


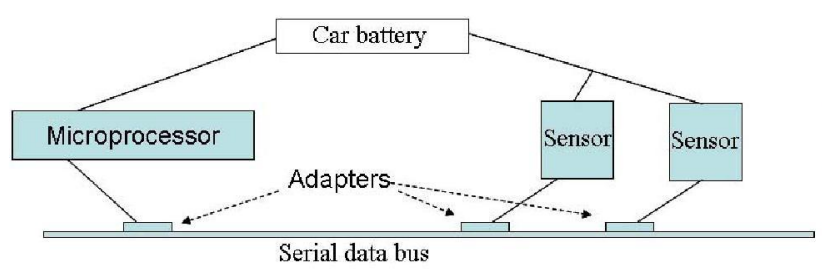

(a) Current Scenario

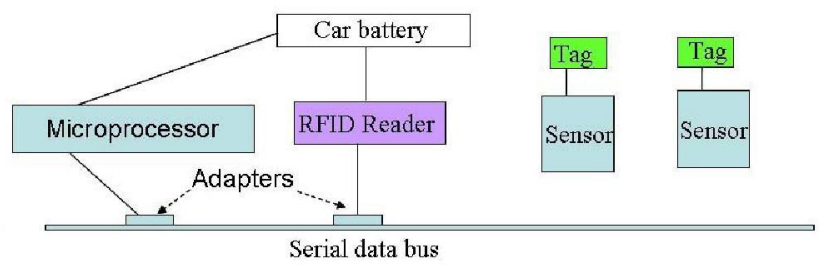

(b) Envisioned Scenario

Fig. 1. (a) In current cars, sensors are connected to power distribution network and serial data bus in the car; (b) in our envisioned scenario, both power wires and communication wires are eliminated by using RFID technology

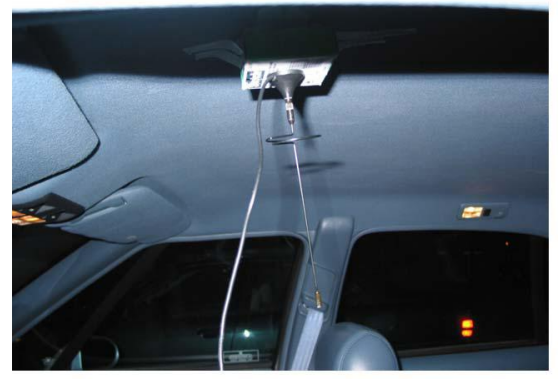

(a) Reader's antenna

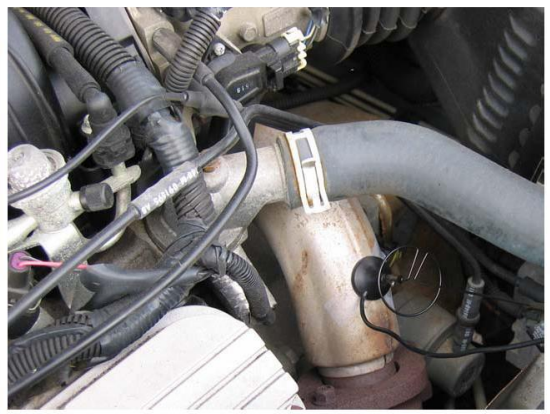

(b) The IE channel

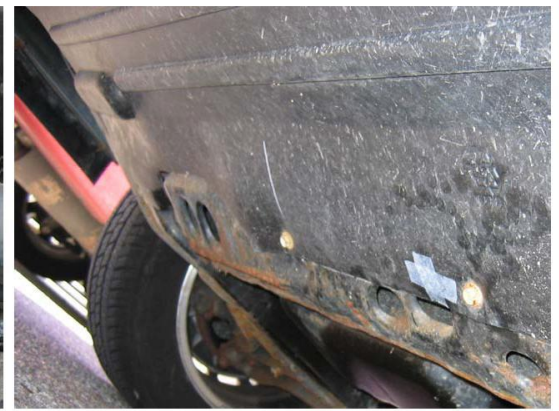

(c) The UE channel

Fig. 2. Various In-car Wireless Channels

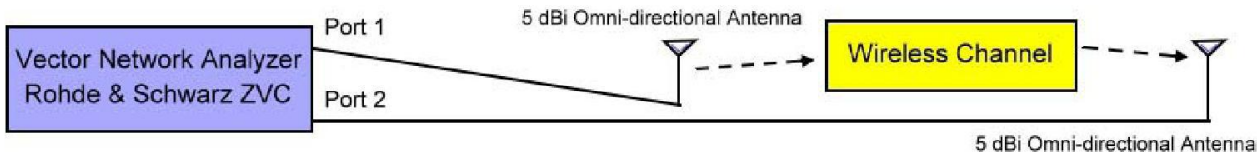

Fig. 3. Block diagram of the experimental setup used for power delay profile measurement.

RMS delay spread. The measurements were repeated several times at each of the locations described in Table II on the campus of Carnegie Mellon University. Except for the $\mathrm{HH}$ data set, in which the car was parked right next to Hamerschlag Hall, all measurements were performed in locations with no nearby buildings. There were no passengers in the car, and some cars and pedestrians were passing by while we performed the measurements.

The results are shown in Table III. Comparing the four channels, the $\mathrm{H}$ channel is the best-case channel since it has almost no obstructions between the transmitting and receiving antennas. The UE channel has a low receiving power, and hence low-power reflections can cause higher RMS delay spread and lower coherence bandwidth. The TR channel is the worst in SH1 and SH3 data set, and this might be due to the reflections caused by various objects put in the trunk. One can also observe that the surrounding buildings and cars do not have much effect on the coherence bandwidth, considering that the reflected power from them is fairly small.

According to [4], the minimum symbol period (Type A Ref-
TABLE I

Parameters Used for Power Delay Profile Measurements

\begin{tabular}{|l|c|}
\hline Parameters & Value \\
\hline \hline Measuring Frequency & $902 \mathrm{MHz}-928 \mathrm{MHz}$ \\
\hline Output Power & $-4 \mathrm{dBm}$ \\
\hline Number of Points (Frequency Sweep) & $401 / 801 / 1601$ points \\
\hline IF Bandwidth & $1 \mathrm{KHz} / 300 \mathrm{~Hz}$ \\
\hline
\end{tabular}

erence Interval, TARI) is $6.25 \mu \mathrm{s}$, and hence the bandwidth of the signal transmitted is about $160 \mathrm{KHz}$. Even with the worstcase channel, the coherence bandwidth is still sufficiently large for the in-car RFID system. This suggests that equalization or channel coding might not be necessary.

\section{B. RFID Transmission Experiment in the Car}

While the coherence bandwidths measured looks very promising, it was also observed that the power loss in the UE channel is more than $80 \mathrm{~dB}$ (Table IV), which is quite large. We designed this second experiment to understand whether the 
TABLE II

Locations of Power Delay Profile MEasurements

\begin{tabular}{|l|c|}
\hline Location & Description \\
\hline \hline HH & Right next to Hamerschlag Hall. No cars within $5 \mathrm{~m}$. \\
\hline SH1 & About $75 \mathrm{~m}$ from Scaife Hall. Several cars parked nearby. \\
\hline SH2 & About $10 \mathrm{~m}$ from Scaife Hall. Several cars parked nearby. \\
\hline SH3 & About $50 \mathrm{~m}$ from Scaife Hall. No cars within $5 \mathrm{~m}$. \\
\hline
\end{tabular}

TABLE III

COHERENCE BANDWIDTHS (MHZ)

\begin{tabular}{|l|c|c|c|c|c|c|c|c|}
\hline Channel & \multicolumn{2}{|c|}{ HH } & \multicolumn{3}{c|}{ SH1 } & SH2 & \multicolumn{2}{c|}{ SH3 } \\
\hline \hline H & 9.79 & 9.89 & N/A & 9.62 & N/A & N/A & 9.27 & 9.29 \\
\hline TR & 8.42 & 8.43 & 9.34 & 7.19 & 5.74 & 8.69 & 5.67 & 6.32 \\
\hline IE & 8.67 & 8.60 & 8.00 & 8.86 & 8.30 & 8.55 & 7.91 & 7.74 \\
\hline UE & 7.20 & 7.55 & 8.08 & 8.02 & 7.75 & 6.88 & 8.49 & 8.09 \\
\hline
\end{tabular}

communication between the off-the-shelf RFID Reader and the Tags is viable in a car environment with large power-loss.

As shown in Figure 4, RFID Tags were placed throughout the engine compartment, as well as on the hood, on the passenger doors, on the front bumper, and under the engine compartment. Surprisingly, with various locations of the antenna of the RFID Reader (in the middle of the passenger compartment, behind the windshield, and on the hood), only the Tags of the closest location (on the hood) could be recognized by the Reader. None of the other Tags could be recognized.

\section{Statistical Analysis}

In this subsection, we evaluate the feasibility of implementing an in-car wireless sensor network from a statistical point of view. To be more specific, we investigate whether the requirements of packet reception rate and maximum packet

TABLE IV

POWER Losses of VARIOUS IN-CAR WIRELESS CHANNELS $(d B)$

\begin{tabular}{|l|c|c|c|}
\hline Symbol Rate & $\mathbf{5 0 0} \mathbf{~ K H z}$ & $\mathbf{1} \mathbf{~ M H z}$ & $\mathbf{2} \mathbf{~ M H z}$ \\
\hline \hline H & 43.83 & 43.86 & 43.94 \\
\hline TR & 60.88 & 60.97 & 60.92 \\
\hline IE & 69.43 & 69.33 & 69.13 \\
\hline UE & 87.50 & 83.81 & 82.01 \\
\hline
\end{tabular}

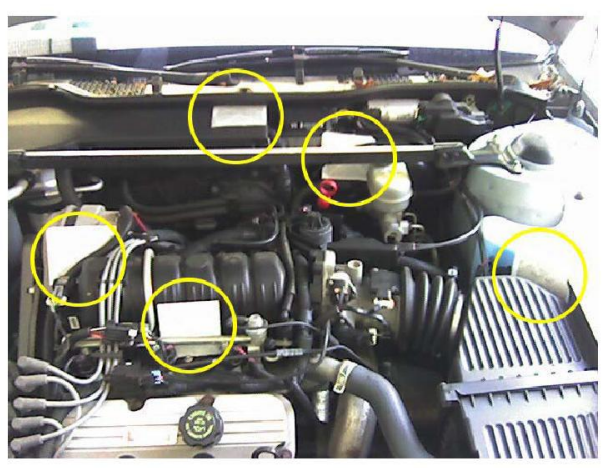

Fig. 4. RFID transmission experiment: the Tags placed in the engine compartment
TABLE $\mathrm{V}$

IN-CAR SENSOR Node SPECIFICATIONS (ASSUMED AND TYPICAL VALUES)

\begin{tabular}{|c|c|c|}
\hline Parameter & Assumed Value & Typical Values \\
\hline \hline Required Pkt Reception Rate & $98 \%$ & Not Available \\
\hline Required Maximum Delay & $500 \mathrm{~ms}$ & $0.016-1000 \mathrm{~ms}$ \\
\hline Packet Size & $125 \mathrm{Bytes}$ & $1-125$ Bytes \\
\hline FEC Correcting capability & $0.1 \%$ & Not Available \\
\hline Noise Power & $-75 \mathrm{dBm}$ & Not Available \\
\hline
\end{tabular}

TABLE VI

The SEtTings of THE Signal Generator

\begin{tabular}{|c|c|}
\hline Parameter & Value/Description \\
\hline Modulation & BPSK \\
\hline Symbol Rate & $500 \mathrm{KHz}$ \\
\hline Center Frequency & $915 \mathrm{MHz}$ \\
\hline Filter & Square-root Raised Cosine $(\alpha=0.1)$ \\
\hline Bit Sequence & Pseudo Random Bit Sequence $(15$ bits $)$ \\
\hline Power Level & $0,-10,-20,-30,-40 \mathrm{dBm}$ \\
\hline
\end{tabular}

delay, which are determined by the specifications of the sensors, can be fulfilled by the statistical characteristics of the in-car wireless channels. Note that we only consider the packet loss and delay contributed by the characteristics of the wireless channels since the physical channel is unchangeable and those contributed by other factors, such as co-channel interference and MAC protocol delay, can be minimized by the MAC and upper-layer protocols. The purpose of this evaluation is to determine whether the idea is feasible even when only the packet loss and delay contributed by the physical channel are considered.

The method to obtain an upper bound on the optimum transmitting power which can satisfy the requirements of the in-car sensors is also shown in this subsection. Table $\mathrm{V}$ lists typical values of the specifications of in-car sensors, as well as the assumed values which are used in the following discussions.

Assuming BPSK is the modulation format used, a bit error rate (BER) of $0.1 \%$ corresponds to a received power level of $-67.665 \mathrm{dBm}^{1}$. In this analysis, we assume that the Forward Error Correction (FEC) code used by the sensor node can correct up to $0.1 \%$ of errors, and hence any received power value larger than $-67.665 \mathrm{dBm}\left(P_{F E C}\right)$ is sufficient. Note that in [5], it has been observed that both the empirical packet reception rate and packet error rate profiles closely follow the theoretical curves in the car environment. It is therefore fairly realistic to use a threshold to determine the packet reception rate or packet error rate.

To calculate the appropriate transmitting power when average received power is given, the pathloss of the channel is needed. The Pathloss (in $\mathrm{dB}$ ), $P L$, is given by $P L=$ $P_{R X}-P_{T X}$, where $P_{R X}$ is the average received power and $P_{T X}$ is the transmitting power.

Figure 5 shows the block diagram representing the experimental setup. The signal generator was configured to transmit

\footnotetext{
${ }^{1}$ The noise is assumed to be AWGN.
} 


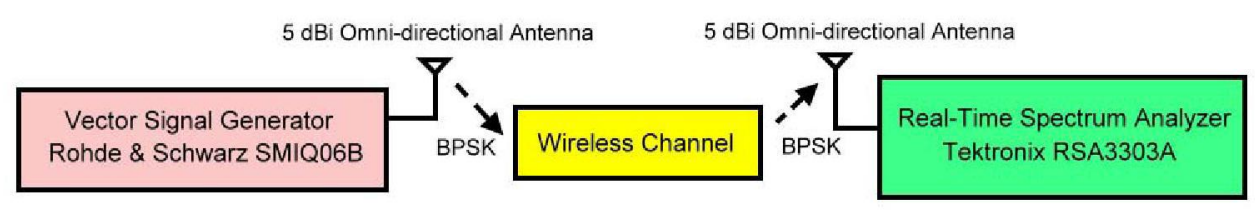

Fig. 5. The block diagram representing the experimental setup

a BPSK signal (see Table VI for the settings of the signal generator) centered at $915 \mathrm{MHz}$. The signal in turn propagates through the connecting cable, the transmitting antenna, the wireless channel, the receiving antenna, the connecting cable, and is finally received by the real-time spectrum analyzer. The experiment was performed in a midsize car parked in a parking lot and with engine turned off. Environmental conditions affecting reception (i.e. cars, pedestrians) were not controlled.

The reason for using a BPSK signal in the experiment is that it is more realistic compared to an impulse signal, i.e., it occupies and utilizes a certain amount of bandwidth. On the other hand, the traditional frequency sweep channel sounding method requires a certain amount of time to sweep through the measuring frequency band and relies on the unverified assumption that the channel coherence time is larger than the time to complete the sweep. Moreover, a BPSK signal is representative in the sense that it is the simplest modulation format and might be suitable for an in-car sensor network with low power consumption requirement. Since most of the sensors have data rates of up to a few hundreds of kilo bits per second, we choose a reasonable value, $500 \mathrm{KHz}^{2}$, as the symbol rate of the BPSK signals in the experiment.

For each transmitting power setting/wireless channel pair, the BPSK signal was transmitted for 5 minutes. The realtime spectrum analyzer recorded the waveform in time domain and arranged it in frames. Blackman window function [6] was applied to each frame and an FFT was performed to convert the data into frequency domain. Then received power was calculated by summing the power values over the $500 \mathrm{KHz}$ bandwidth centered at $915 \mathrm{MHz}$. The final data we got is a vector of received power values representing the received power over this 5-minute period.

1) Packet Reception Rate: One can utilize the results of the fade proportion, i.e., the proportion of time in which received power is below a certain threshold, to obtain an upper bound on the transmitting power which satisfies the packet reception rate requirement. For example, for the IE channel one can conclude that the probability that the received power is less than $1.5 \mathrm{~dB}$ below the average received power is less than $2 \%$. Hence, to achieve $98 \%$ packet reception rate, the average received power has to be at least $P_{F E C}+1.5=-67.665+$ $1.5=-66.165 \mathrm{dBm}$ so that more than $98 \%$ of the time the

\footnotetext{
${ }^{2}$ The data rate for an individual sensor in current cars ranges from $1 \mathrm{Kbit} / \mathrm{s}$ to $100 \mathrm{Kbit} / \mathrm{s}$. Since the bandwidth is shared by all sensors in the car, 500 $\mathrm{Kbit} / \mathrm{s}$ seems to be a reasonable data rate for an in-car wireless sensor node. Hence, the symbol rate of the BPSK signal should be $500 \frac{\mathrm{Kbits}}{\mathrm{s}} / 1 \frac{\mathrm{bit}}{\mathrm{symbol}}=$ $500 \frac{K s y m b o l s}{s}$. UHF Passive RFID technology, which is one of the options for in-car wireless sensor network, also uses $500 \mathrm{KHz}$ of bandwidth [4]
}

received power is larger than $P_{F E C}$. The pathloss of the $\mathrm{IE}$ channel is given by $P L=P_{R X}-P_{T X}=-74.11-(-10)=$ $64.11 \mathrm{~dB}^{3}$. The transmitting power can then be determined by $P_{T X}=P_{R X}-P L=-69.165-(-64.11)=-2.055 \mathrm{dBm}$. Inspection of Figure 6 reveals that there is sufficient evidence in our analysis to show that the in-car wireless channels can support $86 \%, 98 \%, 99 \%$, and $90 \%$ of packet reception rate for the $\mathrm{H}$, the TR, the IE, and the UE channels, respectively, with less than $2 \mathrm{~dB}$ of additional transmitting power. This shows that the TR and the IE channels are good enough to support most of the in-car sensor applications while more experimental data is needed for the $\mathrm{H}$ and the UE channels to have a smaller confidence interval and obtain a bound of higher packet reception rate.

A passive RFID tag has a requirement of having the received power higher than the "chip sensitivity" threshold, which is the minimum received RF power required to turn on the chip inside the passive RFID tag. Chip sensitivity is primarily determined by RF front end architecture and fabrication process [7][8]. The typical value of the chip sensitivity of UHF passive RFID tags is -15 to $-25 \mathrm{dBm}$ [9], which is much higher than $P_{F E C}$, determined by the FEC code error-correcting capability. Hence, the chip sensitivity dominates $P_{F E C}$ and should be used to obtain an upper bound on the optimum transmitting power with the same method described previously. Note that the obtained bound will be loose and too pessimistic. The reason is that the required average received power is much higher than the average received power of our selected data set, which is used for the fade proportion analysis. When transmitting power is higher, the fade proportion plot is more concentrated and represents a better channel condition. The optimum transmitting power is lower in this case. To obtain a tighter bound, additional experimental data measured with higher transmitting power will be needed.

2) Maximum Packet Delay: Average Fade Duration (AFD) corresponds to the average of the time duration in which received power continuously stays below a threshold [10], which can be interpreted as the time that the sensor node stays in continuous outage, i.e., packet delay caused by the wireless channel. In Figure 7, we establish the upper bounds of the 95\% and $99 \%$ confidence intervals of the AFD with the assumption that each fade duration value is independent of others. We can utilize the obtained AFD statistics to quantify the maximum packet delay, which is determined by the specifications of the in-car sensors and is defined as the maximum packet delay that

\footnotetext{
${ }^{3}$ Based on experimental data, the average received power, $P_{R X}$, for the IE channel is $-74.11 \mathrm{dBm}$.
} 


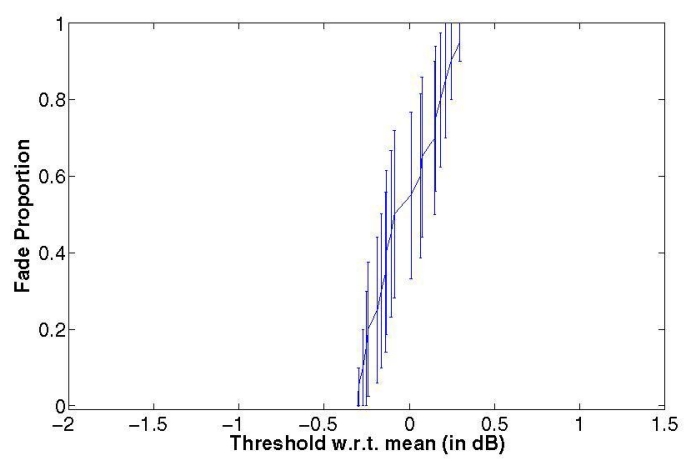

(a) $\mathrm{H}, \mathrm{TX}$ Pwr $=-30 \mathrm{dBm}$

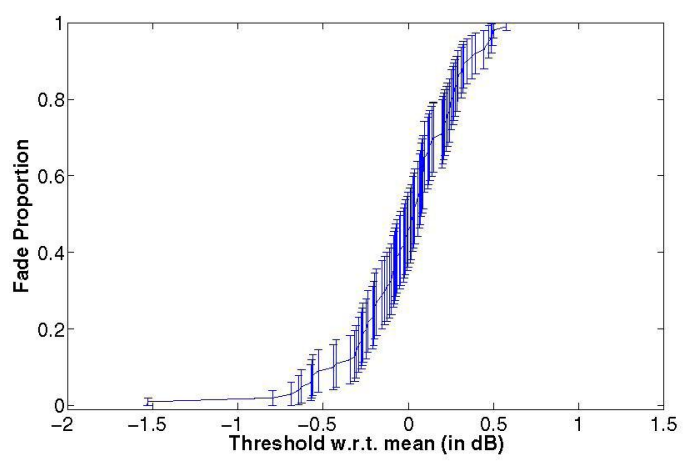

(c) IE, TX Pwr $=-10 \mathrm{dBm}$

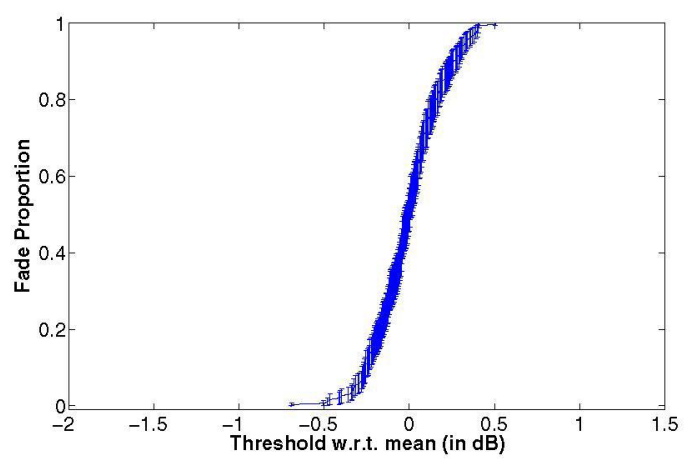

(b) TR, TX Pwr $=-20 \mathrm{dBm}$

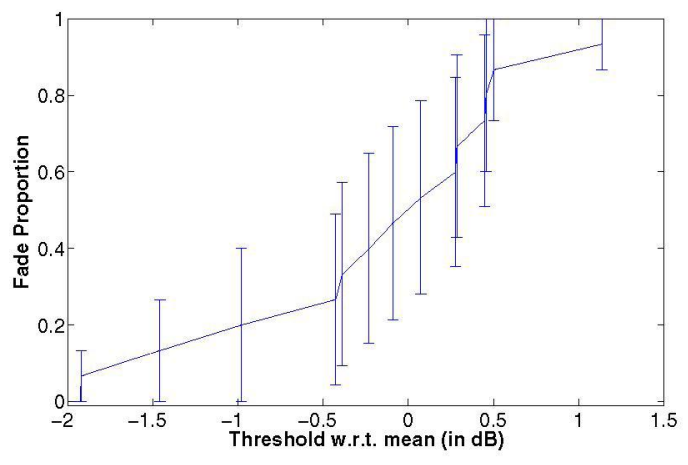

(d) UE, TX Pwr $=0 \mathrm{dBm}$

Fig. 6. Fade proportion statistics

the in-car microprocessor can tolerate for a particular sensor.

Here we use the UE channel as an example to illustrate the method to obtain the upper bound of the optimum transmitting power. Figure 7(d) shows that the received power can stay lower than $1.5 \mathrm{~dB}$ below the average received power for up to $500 \mathrm{~ms}$. The average received power needs to be at least $1.5 \mathrm{~dB}$ higher than $P_{F E C}$ so that the maximum packet delay, i.e., the time that the received power continuously stays below $P_{F E C}$, can be smaller than the maximum packet delay requirement, $500 \mathrm{~ms}$. The average received power is given by $P_{R X}=P_{F E C}+1.5=-67.665+1.5=-66.165$ $\mathrm{dBm}$. The pathloss of the UE channel is given by $P L=$ $P_{R X}-P_{T X}=-67.31-0=-67.31 \mathrm{~dB}^{4}$. Finally, the transmitting power can be determined by $P_{T X}=P_{R X}+P L=$ $-66.165-(-67.31)=1.145 \mathrm{dBm}$.

In Figure 7 , the smallest threshold value corresponds to the smallest fade duration value, which is also the smallest maximum packet delay value that can be satisfied by each channel. Hence, one can conclude that there is sufficient evidence to show that the $\mathrm{H}, \mathrm{TR}, \mathrm{IE}$, and UE channels can

\footnotetext{
${ }^{4}$ Based on experimental data, the average received power, $P_{R X}$, for the UE channel is $-67.31 \mathrm{dBm}$.
}

support maximum packet delays of 130, 170, 310, and 360 $\mathrm{ms}$, respectively. To summarize, the maximum packet delay requirements of most in-car sensors can be satisfied at the expense of a small amount of additional transmitting power.

\section{DISCUSSION}

The results of the experiments reported in section III suggest that, if one uses the antenna of a regular Reader, then most of the Tags placed throughout the car may not get enough energy to be "powered up", thus making them useless in terms of reporting the sensed data by the sensor they are connected to. In the second experiment (subsection III-B) we have used literally the most powerful directional antenna that can fit into the car while in the first measurement (subsection III-A) we used less powerful omni-directional antennas. This suggests that the problem of large power loss cannot be resolved by using powerful, directional antennas. lem:

We propose the following options to circumvent this prob-

a) Use the power distribution network inside the car as a distributed antenna system: The power distribution network already exists in current cars and spreads to various locations inside the car. Therefore, the power loss of the channel 


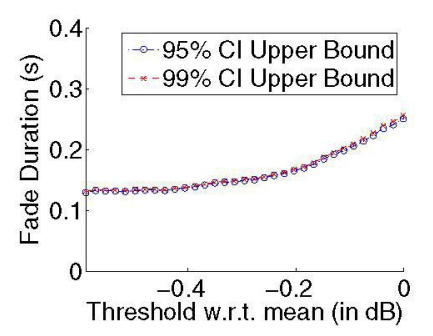

(a) $\mathrm{H}, \mathrm{TX} \mathrm{Pwr}=-30 \mathrm{dBm}$

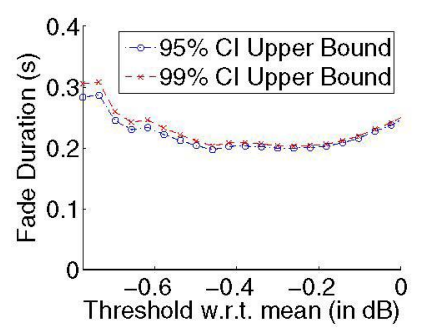

(c) IE, TX Pwr $=-10 \mathrm{dBm}$

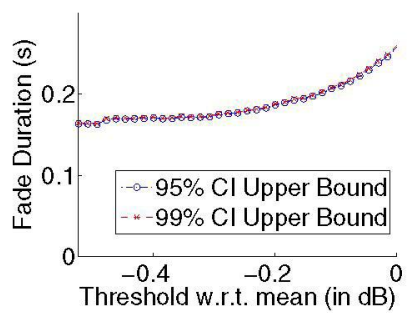

(b) TR, TX Pwr $=-20 \mathrm{dBm}$

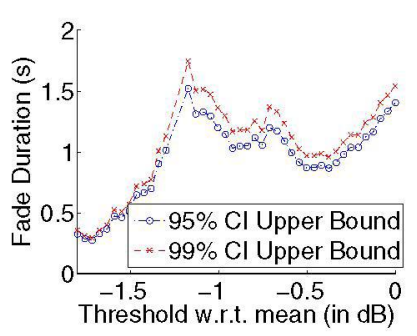

(d) UE, TX Pwr $=0 \mathrm{dBm}$
Fig. 7. Upper bound of $95 \%$ and $99 \%$ confidence intervals of fade duration. Note that the plots do not have a common range on the $\mathrm{x}$-axis or the $\mathrm{y}$-axis due to source data availability and legibility concerns.

between the power distribution network, i.e., the antenna of the base station, and the sensor nodes could be significantly smaller. Further research is needed to better understand how such a distributed antenna system can be implemented [11].

b) Hybrid Architecture: Instead of an all-wireless sensor network, it is possible to implement a hybrid sensor network where some sensors are accessed wirelessly while other sensors which have large power loss are accessed via wires. The number of wireless sensors could still be sufficiently large to have an impact on the weight or the cost of the car and could further increase due to a lower chip sensitivity threshold in the future (as a result of the advance of RF front end architecture and fabrication process).

c) Multi-Reader/Antenna System: The power loss could be decreased if we put multiple RFID Readers/antennas to locations which are closer to the problematic sensor nodes, such as the ones in/under the engine compartment. This approach can also utilize the spectrum more efficiently as different Reader might be able to transmit/receive at the same time and provide better scalibility. The down side is the increased cost of the additional Readers/antennas.

d) Active RFID: Another option is to use active RFID nodes, i.e., nodes with internal batteries, for the locations with large power loss. This eliminates the need of having received power larger than the chip receive sensitivity. The down side of this approach is the increased cost of active RFID nodes and the short battery life.

In terms of reliability, the conducted studies show that TR and the IE channels can support at least $98 \%$ packet reception rate of the sensors while all the 4 in-car wireless channels considered can support the required maximum packet delay of less than $500 \mathrm{~ms}$. The statistical results obtained in this study have important implications in terms of the feasibility of a wireless sensor network in cars: depending on the level of reliability targeted for different applications, they show which sensors can be accessed wirelessly and for which sensors this might be more troublesome. This also suggests the use of stronger FEC and/or transmit power and/or automatic repeat request $(A R Q)$ techniques for higher communication reliability.

\section{CONClusion}

In this paper, we report the use of RFID technology for eliminating the wires that connect sensors to the control unit (microprocessors) in modern cars. To build such an intra-car wireless sensor network, RFID appears to be an interesting option. The major advantage of passive RFID is its low-cost, commercially-available components, and its passive nature among others. The experimental results show promising results in terms of the coherence bandwidth and the reliability of the in-car wireless channels. On the down side, the power loss between a Reader and RFID Tags placed in certain locations (under/in the engine compartment) seems prohibitively large. The power loss problem might be circumvented by using the power distribution network as a distributed antenna system, a hybrid architecture, a multi-Reader/antenna system, or by active RFID nodes. Further research is needed to study the performance and the tradeoffs involved in these options.

\section{REFERENCES}

[1] G. Leen, D. Heffernan, and A. Dunne, "Digital networks in the automotive vehicle," Computing and Control Engineering Journal, vol. 10, no. 6 , pp. 257 - 266, December 1999.

[2] G. Leen and D. Heffernan, "Vehicles without wires," Computing and Control Engineering Journal, vol. 12, no. 5, pp. 205 - 211, October 2001.

[3] T. S. Rappaport, Wireless Communications. Principles \& Practice, 2nd Edition. Upper Saddle River, NJ, USA: Prentice-Hall, 2002.

[4] EPC Radio-Frequency Identity Protocols Class-1 Generation-2 UHF RFID Protocol for Communications at $860 \mathrm{MHz}-960 \mathrm{MHz}$ Version 1.0.9. EPCglobal Inc., January 2005, http://www.epcglobalinc.org.

[5] H.-M. Tsai, C. Saraydar, T. Talty, M. Ames, A. MacDonald, and O. K. Tonguz, "ZigBee-based intra-car wireless sensor network," in Proc. IEEE International Conference on Communications (ICC 2007), June 2007.

[6] A. V. Oppenheim, R. W. Schafer, and J. R. Buck, Discrete-Time Signal Processing. Prentice Hall, 1999.

[7] P. V. Nikitin and K. V. S. Rao, "Performance limitations of passive UHF RFID systems," in IEEE Antennas and Propagation Symposium, July 2006.

[8] G. D. Vita and G. Iannaccone, "Design criteria for the RF section of UHF and microwave passive RFID transponders," IEEE Transactions on Microwave Theory and Techniques, vol. 53, no. 9, pp. 2978-2990, September 2005.

[9] O. K. Tonguz and H.-M. Tsai, "New channel measurements for GM RFID project," Carnegie Mellon University, Tech. Rep. GM-3-11-2005, November 2005.

[10] H. Hashemi, M. McGuire, T. Vlasschaert, and D. Tholl, "Measurement and modeling of temporal variations of the indoor radio propagation channel," IEEE Trans. Veh. Technol., vol. 43, no. 3, pp. 733-737, August 1994.

[11] S. R. Saunders, Antennas and Propagation for Wireless Communication Systems. John Wiley and Sons, 1999. 